Herausforderungen der Transformation

\title{
Kybernetische Governance als Planwirtschaft 2.0?
}

\author{
Eine gesellschaftliche Transformation setzt \\ eine neue Form des Wirtschaftens voraus.
} Wie diese Wirtschaftsweise aussehen kann, wird zunehmend von den unterschiedlichen Aspekten der Digitalisierung beeinflusst. Aufgrund des disruptiven Charakters der Digitalisierung wird zunehmend über eine Neuinterpretation von Planung und Planwirtschaft diskutiert. Von Ulrich Petschow

D ie Diskussion um „planetare Grenzen“ verweist auf die Notwendigkeit neuer Formen der Bewirtschaftung von Quellen und Senken. Der Wissenschaftliche Beirat der Bundesregierung Globale Umweltveränderungen (WBGU) hat dies exemplarisch am Beispiel des Budgetansatzes verdeutlicht. In Anbetracht der Emissionen klimarelevanter Gase bedeutet dies, dass in einem eher kurzen Zeitraum erhebliche Emissionsreduktionen erforderlich sind. Gegenwärtig ist weiterhin eher offen, wie diese Emissionsreduktionen in der Praxis realisiert werden sollen, da weitreichende Eingriffe in die Wirtschaftsund Lebensweise erforderlich sind und gegenwärtig nicht absehbar ist, wie dies gelingen kann. Vielfältige Diskurse existieren dazu, wie die Rahmenbedingungen zu gestalten sind, die ein nachhaltiges Wirtschaften ermöglichen sollen. Des Weiteren werden grundlegende Veränderungen des Gesellschaftssystems sowie soziale und technologische Innovationen eingefordert, nudging wird zunehmend als relevant angesehen.

Mithin existieren Pläne, welche Reduktionen in welchen Zeiträumen zu erreichen sind, doch wie diese Pfade zu beschreiten sind, ist offen und letztlich ungeplant. Die Vorstellungen gehen im Wesentlichen von Kontextsteuerungen aus, indem die Obergrenzen letztlich an Markt und Gesellschaft durch geeignete Rahmenbedingungen vermittelt werden müssen.

\section{Veränderungen des „Betriebssystems“}

In den vergangenen Jahren haben sich zudem die gesellschaftlichen (Steuerungs-)Strukturen umfassend verändert, indem es zu umfassenden Vernetzungen, sowohl der Produktion als auch der Individuen, gekommen ist. Ersteres wird unter dem Motto "Internet of Things“ oder auch Industrie 4.0 diskutiert, letzteres im Rahmen der vielfältigen sozialen Netzwerke. Vereinfacht ausgedrückt finden Austauschprozesse, auch zwi- schen Mensch und Natur auf Märkten und unter der Mediation von Algorithmen, statt. Entwicklungen im Bereich Big Data und Artificial Intelligence (AI) werden massiv vorangetrieben und verändern in der Perspektive das „Betriebssystem“ der Gesellschaften. Ganz konkret bedeutet das, dass sich neue Steuerungsformen herausbilden, die gegenwärtig überwiegend von privatwirtschaftlich organisierten (sozialen, Handels-und Produktions-)Plattformen gestützt werden. Es werden zunehmend disaggregierte Daten in sehr hoher individueller Auflösung mit zeitlicher Entwicklung generiert, die es zunehmend als realistisch erscheinen lassen, dass prädiktive Aussagen generiert werden können. [1]

Mithin haben wir es mit einem zunehmend dynamischen System zu tun, in welchem große Plattformen wie Amazon oder Alibaba zunehmend ,jenseits des Marktes“ agieren und die Rolle der Planung an Relevanz gewinnt. Es ergeben sich neue Formen sozialer und gesellschaftlicher Steuerung, in denen der Frage der Nachhaltigkeit bislang nur begrenzt Relevanz beigemessen wurde. Ausgangspunkt dieses Beitrags war einerseits die Diskussion um die Plattformökonomien und deren Infrastrukturen und andererseits ein Artikel von chinesischen Wissenschaftlern, die die Frage nach einer Planwirtschaft neu gestellt haben.

\section{Plattformökonomien und eine neue Planwirtschaft}

In jüngerer Zeit sind Überlegungen angestellt worden, die vor dem Hintergrund von Big Data und Artificial Intelligence (AI) darauf hinweisen, dass sich Märkte grundsätzlich verändern, und damit einhergehend auch das Zusammenspiel von Angebot und Nachfrage. Mithin wird auch über eine Neuinterpretation von Planung und Planwirtschaft diskutiert. Der Austausch findet zunehmend weniger auf anonymisierten Märkten statt. Die Relevanz der (Online-)Plattformen nimmt deutlich zu und sie verändern die Art des Austausches und die Kontexte (Datenverfügbarkeit und -aggregation, algorithmische Steuerung) deutlich. Des Weiteren werden zunehmend Bemühungen unternommen, durch die auf den Plattformen generierten Daten Wissen über künftige Nachfrage zu generieren.

In einem Artikel haben die Autoren Wang und Li (2017) dargelegt, inwieweit die Planwirtschaft in der Ära von Big Data wieder an Relevanz gewinnen könnte. Dabei verweisen sie auf die Diskussionen der dreißiger Jahre des letzten Jahrhunderts um Oskar Lange und die „socialist calculation“, die die Ver- 
bindung von zentraler Planung und Marktsimulationen diskutierte. Die beiden Autoren entwickeln die folgende Vorstellung: „big data as technical condition, platform economy as institutional und organizational conditions, to forming the big data bases and state-owned enterprises leading operated internet platform economy in the field of competition, which can achieve to make the market play a decisive role in the allocation of resources and the state play the leading role $[. . .]^{\text {“ }}$

Knapp zusammengefasst sind es drei Argumente:

I Big Data ermöglicht es, sogenanntes „tacit knowledge“, das in der Gesellschaft weit verstreut ist, zu finden und zu nutzen.

- Big Data kann die Vorausschau verbessern und das Problem der zeitlichen Verzögerung zwischen Plan und Umsetzung (Echtzeit) überwinden.

- Big Data kann ebenso dazu beitragen, die Skaleneffekte der Massenproduktion zu erschließen und gleichzeitig den „long tail“, also personalisierte und diversifizierte Nachfrage und Angebot, zusammenbringen. Mit anderen Worten geht es ihnen darum, den Markt in Echtzeit besser zu steuern.

Die Autoren des Artikels verweisen darauf, dass Onlineplattform-Monopole letztlich Zentralplanungseinrichtungen ähneln würden. Es wäre demnach sinnvoll, dass der Staat, eine supermonopolistische Plattform (als Infrastruktur) etabliert, die die Grundlage für die Geschäftsaktivitäten von Unternehmen darstellen sollte. Im Grundsatz zielt dies darauf ab, dass die Infrastrukturen staatlich bereitgestellt werden sollten, ein Vorschlag, der, vor dem Hintergrund der Machtkonzentration der Internetkonzerne, auch von anderen Akteur/innen eingebracht wird. [2]

Gegen diese Vorstellungen werden in der Regel zwei Einwände vorgebracht: Zum einen wird darauf verwiesen, dass die umfassende Generierung und das Sammeln von Daten das eine sind, dass aber die „sensible“ Nutzung dieser Daten eine ganz andere Sache ist, insbesondere wenn staatliche Akteur/ innen einbezogen werden. Zum anderen wird gefragt, inwieweit eine solche Ökonomie überhaupt in der Lage ist, Innovationen zu generieren.

Nun ist es zweifelsohne so, dass realsozialistische Planwirtschaft letztlich gescheitert ist. Auf einer übergeordneten Ebene geht Harari (2017) davon aus, dass der Niedergang im Wesentlichen darin begründet lag, dass „using organised economics and dictatorial government for processing data" zu Fehlsteuerungen führte. Der Kapitalismus war demnach erfolgreich aufgrund des "distributed processing of data“. Mithin wird auf das von Hayek aufgeworfene Problem Informationsgenerierung verwiesen.

\section{Ein Blick zurück: Cybersyn - (de-)zentrale Steuerung der Volkswirtschaft}

Die Idee der Kybernetik, also der Wissenschaft der Steuerung und Regelung von Systemen (Maschinen, sozialen Organisationen und lebenden Organismen), wurde unter anderem von Wiener in den dreißiger und vierziger Jahren des letzten
Jahrhunderts entwickelt und hat insbesondere in den fünfziger und sechziger Jahren viele Wissenschaftsbereiche beeinflusst („Macy Konferenzen“, Pias 2003). In den sechziger Jahren wurde die Kybernetik als Heilsbringer verstanden und als Lösungsansatz für vielfältige gesellschaftliche Problemlagen, unter anderem auch die Frage ökonomischer Steuerung, angesehen. Der Ansatz wurde auch in der damaligen DDR und UdSSR zumindest auf der wissenschaftlichen Ebene aufgegriffen. [3] Kybernetik war angetreten, eine Einheit der Wissenschaften zu erreichen, allerdings „entstand keine Einheitswissenschaft, die Lösungen für Steuerungsprobleme aller Art zur Verfügung stellen würde“. Heute finden sich vielfältige Bezüge zu der Kybernetik in den Systemwissenschaften, aber auch in den Ansätzen von Big Data und AI (vgl. Oelkers 2008).

\section{Chile und Stafford Beer}

Einer der wichtigen Autoren einer sich herausbildenden Managementkybernetik war Stafford Beer, der ein organisationales Leitbild (,viable system model“) entwickelt hatte, das sich an das menschliche Nervensystem anlehnen sollte und, so die Meinung von Beer, letztlich für alle resilienten Organisationen relevant wäre. Mithin ein System, das „survives. It coheres, it is integral" und gleichwohl Mechanismen und Möglichkeiten hat "to grow and learn, to evolve and adapt“ (Beer 1972, S. 583).

Beim „viable system model“ ging es nicht um ein zentralisiertes Managementsystem, vielmehr sollte es in „decentralizing, worker participative and anti-bureaucratic manner" gestaltet sein (Beer 1972, S. 257). Mithin verdeutlicht der Ansatz, dass es nicht um ein rein technokratisches Vorhaben ging, also nicht um ein technisches Vorhaben um die Ökonomie zu steuern, sondern dass es auch um die Gestaltung durch neue Steuerungsansätze und durch "revolutionäres Computing" ging (Medina 2006, S. 574). Außerdem wurde das Spannungsverhältnis von Zentralisierung und Dezentralisierung angesprochen, ebenso wie die Frage der Technikgestaltung.

Als Anfang der siebziger Jahre in Chile der Sozialist Salvador Allende gewählt wurde, kam es zu Verstaatlichungen von zentralen chilenischen Unternehmen und es stellte sich die Frage, wie dieser neue Bereich planvoll gesteuert werden könnte. Stafford Beer wurde eingeladen, seine kybernetischen Vorstellungen des Managements in Chile umzusetzen. Das Projekt Cybersyn (mit vielfältigen Teilprojekten) sollte eine real-time Steuerung der Wirtschaft ermöglichen. Dabei sollten sowohl die Produktions- als auch die Nachfragestrukturen identifiziert und $\mathrm{zu}$ einem Abgleich gebracht werden. Die zentrale Steuerung der Produktion (wie auch das Regieren selbst) sollte in der Vision mit der elektronischen Erfassung der Wünsche bzw. der potenziellen Nachfrage verbunden werden.

Dazu wurde ein kybernetisches Fabrikmodell entwickelt und zugleich ein Datennetzwerk für die schnelle Übertragung ökonomischer Daten zwischen der Planungsinstanz und den Fabriken geschaffen. Für Cybersyn stand ein Großrechner zur 
Verfügung, für die Informationsbereitstellung aus den Unternehmen des verstaatlichten Sektors wurde ein Telexnetzwerk aufgebaut und damit ein nationales Kommunikationsnetzwerk geschaffen. Dieses System sollte eine Realtime-Kontrolle der Ökonomie ermöglichen. In der Praxis war es allerdings nur möglich, die Daten einmal am Tag zu übertragen (über das Cybernet). Zudem existierte ein Simulationsmodell auf der Basis des Dynamo-Compilers (Forrester 1972), einer kybernetische Programmiersprache für Computersimulationen, um die Wechselwirkungen zwischen Faktoren und deren Einfluss auf die Dynamik eines Systems zu erforschen. [4]

Im Gegensatz zu der Zentralplanung der Sowjetunion, ging Allende mit seiner Interpretation des Sozialismus davon aus, dass durch eine dezentrale Governance beispielsweise auch Mitarbeiter/innenbeteiligung und insbesondere die individuellen Freiheiten unterstützt werden sollten. Allerdings spielten in Allendes Interpretation des demokratischen Sozialismus Spannungsverhältnisse zwischen Dezentralität und Zentralität eine entscheidende Rolle. Ein zentraler datentechnisch vernetzter „Oops room“ [5] sollte eine neue Umgebung für eine eher zentrale Entscheidungsfindung generieren. Diese zentrale Schaltstelle wurde allerdings nie in Betrieb genommen, gleichwohl wurde er zum Symbol des Projektes.

In der Summe hatte das Cybersyn Projekt letztlich nicht die Fähigkeiten, die Transformation der chilenischen Ökonomie vom Kapitalismus zum Sozialismus hinreichend zu unterstützen. Vielmehr hatte das Vorhaben selbst mit dem Aufbau der Strukturen und den täglichen Operationen ausreichend zu tun. Mithin wurden mit Cybersyn eine Vielfalt von Ideen generiert und weiterentwickelt, deren rechentechnische Basis allerdings den Anforderungen der Transformation bei Weitem nicht genügen konnten.

Morozow (2014) verweist darauf, dass das Projekt Cybersyn auch als Vorschau auf die Zukunft verstanden werden kann. Die wirtschaftskybernetischen Ideen von Beer stellen demnach "eine zentrale Denke“, sowohl in Wirtschaftspublikationen als auch auf Technologiekonferenzen, dar und ermöglichen eine Vorstellung einer dynamischen Echtzeitplanung für Unternehmen im Sinne der Gewinnmehrung. Die grundlegenderen gesellschaftsgestaltenden Ideen von Beer haben hingegen nicht den Weg in den Mainstream gefunden.

\section{Informationsgenerierung und -verarbeitung}

Ein zentraler Aspekt der Diskussion um Plan und Markt zielt auf die Generierung von Informationen beziehungsweise Wissen ab. Hayek hat darauf hingewiesen, dass Planwirtschaften letztlich am Informationsproblem scheitern, da sie unter anderem nicht in der Lage sind, lokales und disperses Wissen zu erfassen.

Der informationsökonomische Ansatz des Marktes als Entdeckungsverfahren von Hayek (1945) unterscheidet sich in starkem Maße von anderen ökonomischen Ansätzen, insbesondere auch von dem Walrasianischen Gleichgewichtsansatz.
Dieser Standardansatz der Ökonomie wurde von Oskar Lange genutzt, der die Debatte um „socialist calculation“ angestoßen hatte. Lange und andere haben argumentiert, dass der Zentralplaner Preise und/oder Mengen setzen kann, um das Marktergebnis so zu beeinflussen, wie es gesellschaftlich wünschenswert sei, indem sie beispielsweise Externalitäten und andere Faktoren einbeziehen, die am (reinen) Markt nicht berücksichtigt würden. Hayek argumentierte dagegen, dass es für den Zentralplaner unmöglich sei, Preise und Mengen richtig auszuwählen, weil die erforderlichen Informationen über Präferenzen nicht im Vorhinein zur Verfügung standen und nur durch den Prozess der Marktinteraktion (Wettbewerb) generiert werden können: „procedure for discovering facts which, if the procedure did not exist, would remain unknown or at least not be used“ (zit. nach Bowles et al. 2017, S. 218).

\section{Überwindung des Informationsproblems Big Data und Al?}

Die dynamische Entwicklung von Rechenkapazitäten und das Wachstum von Onlinetransaktion und -kommunikation haben das Sammeln und die Verarbeitung von großen Datenmengen möglich gemacht. Viele Plattformen und Onlinemärkte beziehen nun Algorithmen für das Preissetting ein, die sie direkt aus menschlichen (Trans-)Aktionen generieren, und werfen damit neue Fragen über den Prozess, durch den Informationen generiert werden, und die Veränderungen von Marktprozessen auf. Die These von Wang und Li besagt, dass mit den neuen technischen Möglichkeiten das Informationsproblem gelöst werden könnte.

Chavalarias (2016) geht mit Blick auf die Generierung von Informationen beziehungsweise auch von Präferenzen noch einen Schritt weiter, indem er auf die Ranking- und Bewertungssysteme im Netz verweist.Diese Plattformen machen Vorschläge (Kaufempfehlungen), die sich einerseits aus den früheren Auswahlentscheidungen speisen und andererseits auch in Beziehung gesetzt werden $\mathrm{zu}$ anderen Nutzer/innen und den sozialen Netzwerken und damit versuchen, Kaufentscheidungen zu beeinflussen. Die internetgestützten sozialen Netzwerke (aber auch die vielfältigen elektronischen "devices“ zum Beispiel Google) haben einerseits zu einer weiten Verbreitung dieser Praktiken geführt und andererseits umfassende Datenquellen $\mathrm{zu}$ individuellem Verhalten generiert. Die Performance von (Kauf-)Empfehlungssystemen und die Möglichkeiten, das Verhalten von Internetusern vorherzusagen, verbessern sich offenbar kontinuierlich, sodass „predictive analytics“ und „predictive shipping“ zunehmend als realistische Optionen angesehen werden. Chavalarias verweist zudem darauf, dass die sozialen Interaktionsprozesse auch dazu führen, dass sich individuelle Präferenzen herausbilden (entgegen der gängigen ökonomischen Ansätze). Experimente konnten nachweisen, dass Settings mit unterschiedliche Ausgangsbedingungen und unterschiedlichen sozialen Interaktionsmöglichkeiten dazu führten, dass Präferenzen sich unterschiedlich 
entwickeln und damit entscheidend auch von den beiden Parametern (Ausgangsbedingungen und sozialen Interaktionsformen) abhängen. Übertragen bedeutet das, dass die (Interaktions-)Kontexte die Herausbildung von Präferenzen befördern und in einer Gesellschaft, in der immer mehr soziale Interaktionen internetgestützt sind, sowohl soziale Bewertungssysteme, aber zunehmend auch Algorithmen die Interaktionen mit beeinflussen.

Mithin wird deutlich, dass durch das Internet neue Formen sozialer Interaktionen realisiert werden, die in der Summe auch weitreichende Verhaltensänderungen mit sich bringen können. In diesem Kontext ist auch auf nudging zu verweisen, wo auch darauf abgezielt wird, Menschen in ihrem Verhalten $\mathrm{zu}$ beeinflussen und zwar in dem Sinne, dass es sowohl ihnen, als auch der Gesellschaft besser gehen möge. Ist der Ansatz des nudging bis dato noch überwiegend offline orientiert, so ist davon auszugehen, dass die sozialen Netzwerke ein ideales weiterzuentwickelndes Spielfeld darstellen werden (Devereaux 2017). [6]

\section{Plattformen: Markt- und Gesellschafts- steuerung}

In den entwickelten Volkswirtschaften hat die durchschnittliche Unternehmensgröße zugenommen, zum Teil derart umfassend, dass das Scheitern von Unternehmen in einzelnen Volkswirtschaften zu makroökonomischen Schocks führen kann. Die plattformgetriebenen Tech-Unternehmen, also beispielsweise Amazon oder Alibaba (mit heute bereits 500 Mio. Nutzer/innen), expandieren nochmals stärker, sodass deutlich wird, dass vielfältige und zunehmende ökonomische Austauschprozesse nicht im anonymen Markt, sondern innerhalb von Unternehmen stattfinden. Auch unter Berücksichtigung neuer vielfach marktorientierter interner Steuerungsprozesse wird deutlich, dass hierarchische Planung einen zunehmenden Stellenwert erreicht und die Relevanz des marktlichen Austausches abnimmt. Zudem verändern sich die Marktinteraktionen. Hierbei wird auf Uber verwiesen, das seinen Fahrer/innen computergestützte Routen vorgibt und automatisierte Preisbildungsprozesse nutzt. Der Markt ist dementsprechend nicht mehr der Ort der Preisbildung, wo Angebot und Nachfrage zusammentreffen, sondern ein Computermodell, eine Simulation von Angebot und Nachfrage, die in Echtzeit angepasst wird.

Neben der Datengenerierung für die Produktion zielen aktuelle Ansätze in China darauf ab, die Gesellschaft selbst zu „steuern“.

In der Volksrepublik China soll ein „social credit system“, das seit dem Jahr 2003 entwickelt wird, nunmehr ab dem Jahr 2020 in der Breite in die Praxis umgesetzt werden (vgl. Pohlmann 2018; Diab 2017). Der „social credit“ soll fünf Dimensionen abbilden und zwar „lawfulness", moral behaviour", social engagement" , ,activities of public interest" und schließlich "environmental protection“. In einer Testregion wurden Bürger/innen mit einem Anfangsbestand von 1.000 Kreditpunkten ausgestattet, je nach ihrem Verhalten wurden Kreditpunkte gewonnen oder auch verloren. Es gibt unterschiedliche Grade von A plus, A, B, C bis schließlich D. Hatten die Bürger mehr als 1.000 Punkte, gehörten sie zur A plus Gruppe. Neben den sozialen und politischen Bewertungen spielen auch umweltrelevante Aspekte eine Rolle: Wohnen in einem großen Apartment oder auch die Fahrt zur Arbeit mit einem (großen) Auto führen zum Punktabzug, wird der Weg zur Arbeit mit dem Fahrrad zurückgelegt, so werden Punkte gutgeschrieben. Dieses Bewertungssystem spielt bereits jetzt in einigen Bereichen eine wichtige Rolle. Alibaba und das Tochterunternehmen Sesam Credit sind in die Pilotphase des sozialen Kreditsystems eingebunden. Dabei werden umfangreiche Datenbestände angelegt und Bewertungen vorgenommen, ebenso werden Verhaltenspräferenzen und das persönliche Netzwerk, in denen sich die Individuen bewegen, miterfasst, und unterschiedlichste Datenbestände zusammengeführt. Die Nutzer/innen können jederzeit online überprüfen, wie sich der Stand ihres Ratings verändert. Sesam Credit verfügt selbst wiederum über eine Vielzahl von Kooperationspartnern, die auf die Informationen zurückgreifen können, dazu gehören auch Onlinedating-Plattformen.

Vor dem Hintergrund der vielen neuen Methoden der Datenerfassung und Verarbeitung ist es möglich, dass die Planwirtschaft in neuer Form wieder auferstehen könnte. Damit kann die Nachfrage effektiv befriedigt werden, während die zentrale Kontrolle von Produktion und Investmentkosten und -gewinnen erreicht werden könnte. Damit könnten auch umweltrelevante Tatbestände in die Kalkulation einbezogen werden. Allerdings gibt es Herausforderungen:

Planung auf der Grundlage der Wissensbasis der Mikroebene heißt auch, weitgehend alle Bedürfnisse der Individuen zu kennen. Dies geht nicht ohne die Ausleuchtung der Privatsphäre. Die Tech-Giganten schaffen mit ihren algorithmischen Steuerungsmodellen jene Planbarkeit, die die Voraussetzung für die Planwirtschaft wäre. Dies bedeutet, dass sowohl diese Form der plattformgestützten Marktwirtschaft als auch eine sozialistische Planwirtschaft letztlich vor den gleichen Dilemmata stehen, nämlich der Notwendigkeit der Verfügbarkeit von detaillierten Wissen über die Individuen.

Mit der Möglichkeit der umfassenden Datenverfügbarkeit und der zunehmend auch möglich werdenden prognostischen Fähigkeiten von Systemen der künstlichen Intelligenz könnten in der Tat im Idealfall einige der "drawbacks“ der Planwirtschaft überwunden werden. Allerdings besteht kein Zweifel daran, dass damit eine erhebliche Machtkonzentration verbunden ist, beispielsweise in der Hand des Staates (oder einer internationalen Organisation). Allerdings ist gleichzeitig festzustellen, dass die gegenwärtigen Tendenzen der Plattformökonomie in eben diese Richtung zielen, indem insbesondere die Tech-Unternehmen in den USA und China über eine unvorstellbare Macht verfügen. Insofern stellt sich sehr wohl die Frage nach der Gestaltung der Plattformen, nicht allein im Sinne von den zwei Pfaden eines digitalen Kapitalismus oder eines digitalen Sozialismus. 


\section{Planung und Steuerung}

Die zunehmende Generierung von Daten, insbesondere auch von umweltrelevanten Daten, ermöglichen mindestens im Grundsatz neue Steuerungsmodelle. Vor dem Hintergrund der weitreichenden Ziele der planetaren Grenzen wird es in jedem Fall der Planung und der Instrumentierung des Übergangs bedürfen. Diese Herausforderungen werden durchaus gesehen, die Betonung auf die Förderung sozialer Innovationen fokussiert darauf, dass Verhaltensänderungen im Sinne einer nachhaltigen Entwicklung auch auf der individuellen Ebene gefördert werden müssen. Gegenwärtig erscheint es allerdings so, dass indirekte Beeinflussungen wie durch nudges durchaus an Relevanz gewinnen, dass aber gegenwärtig die Relevanz der Steuerung durch Plattformökonomie und soziale Netzwerke im Wesentlichen von Großkonzernen wahrgenommen und in ihrem Sinne genutzt wird. Es findet mithin eine Gesellschafts- und Verhaltenssteuerung statt, die allerdings wenig zu einer nachhaltigen Entwicklung beiträgt beziehungsweise beitragen kann. Übernimmt man die These, dass das Informationsproblem, auf das Hayek verwiesen hat, mit den neuen Technologien weitgehend einer Lösung zugeführt werden kann, so werden neue Ansätze zur Umsetzung der Pläne, beispielsweise der Dekarbonisierung, dringend erforderlich, die eben auch gerade die vielfältigen Vernetzungen nutzen sollten. Es wären smarte, selbststeuernde Systeme denkbar, die Umweltherausforderungen in Entscheidungsprozesse systematisch einbeziehen und damit selbst zur Pfadgestaltung beitragen können.

Unklar ist allerdings, wie dystopische Ansätze (Matrix), wie sie in China vorangetrieben werden sollen, letztlich vermieden werden können. Dies geschieht im Übrigen auch unabhängig davon, ob es sich um zentralstaatliche Ansätze oder Ansätze der monopolartigen Hightech-Industrien handelt. Insofern wird es sehr darauf ankommen, Systemarchitekturen so zu gestalten, dass der Wandel unterstützt und gleichzeitig Machtkonzentration gemindert wird. Die Vorstellungen Stafford Beers zur Systemgestaltung, aber auch zu Machtstrukturen, können dazu Orientierungen geben.

\section{Anmerkungen}

[1] Neben den Datenbeständen der Tech Unternehmen sind auch diejenigen der Geheimdienste wie der NSA zu erwähnen (vgl. Klimburg (2017).

[2] Die Idee von Hightech-Planungssystemen wird auch von anderen Autor/innen vorgebracht, wie Mason (2016) oder Frase (2016). Allerdings sehen die chinesischen Autoren die mit diesen Überlegungen verbundene Überwachung als notwendige Voraussetzung für ein solches System an.

[3] Hörl und Hagner verweisen darauf, dass „Kybernetik dabei nicht nur die Chiffre für den technischen Traum totaler Kontrolle (war), sondern [...] auch zum Einfallstor für Konzepte der Selbstorganisation (wurde) “ (Hörl et al. 2005, S. 35).

[4] Die Modellierungen für die Publikation von Meadows et. al. (1972) "Grenzen des Wachstums" stützten sich ebenfalls auf Forrester.

[5] www.proyectoidis.org/cybersyn-sinergia-cibernetica/
[6] Die aktuelle Diskussionen um Facebook und Cambridge Analytics verweisen darauf, dass die Möglichkeiten der Beeinflussung bereits im Heute genutzt werden. Über die Wirksamkeit dieser Ansätze lässt sich gegenwärtig allerdings trefflich streiten.

\section{Literatur}

Beer, S. (1972): Brain of the Firm: A Development in Management Cybernetics. New York. Herder and Herder.

Bowles, S./Kirman, A./Sethi, R (2017): Retrospectives - Friedrich Hayek and the Market Algorithm. In: Journal of Economic Perspectives 31/2: 215-230.

Chavalarias D. (2016): The unlikely encounter between von Foerster and Snowden: When second-order cybernetics sheds light on societal impacts of Big Data. In: In: Big Data and Society: 1-11.

Devereaux, A, (2017). The Nudge Wars: a modern Socialist Calculation Debate. GMU Working Paper in Economics No. 17-44. George Mason University Department of Economics.

Diab, R. S. (2017): Becoming Infrastructure: Datafication, Deactivation, and the Social Credit System. In: Journal of Critical Library and Information Studies 1/1.

Forrester J. W. (1972): Grundzüge einer Systemtheorie. Wiesbaden, Gabler.

Frase, P. (2016): Four Futures. Life After Capitalism. London, Verso.

Harari, Y. N. (2017): Homo Deus: A Brief History of Tomorrow. New York, HarperCollins.

Hayek, F. A.v. (1945): The Use of Knowledge in Society. In: AER 35/4: 519-530.

Hörl, E./Hagner, M. (2005) (Hrsg.): Die Transformation des Humanen. Beiträge zur Kulturgeschichte der Kybernetik. Frankfurt am Main, Suhrkamp Verlag.

Klimburg, A. (2017): The Darkening Web: The War for Cyberspace. New York, Penguin.

Mason, P. (2016): Postkapitalismus: Grundrisse einer kommenden Ökonomie. Berlin, Suhrkamp.

Meadows D. et al. (1972): Die Grenzen des Wachstums. Stuttgart, DVA. Medina, E. (2006): Designing Freedom, Regulating a Nation: Socialist Cybernetics in Allende's Chile*, In: J. Lat. Amer. Stud. 38: 571-606.

Morozov, E, (2014): The Planning Machine. Project Cybersyn and the Origins of the Big Data Nation. The New Yorker 13. 10. 2014.

Oelkers, J. (2008): Kybernetische Pädagogik: Eine Episode oder ein Versuch zur falschen Zeit? In: Hörl, E./Hagner, M. (2005) (Hrsg.): Die Transformation des Humanen - Beiträge zur Kulturgeschichte der Kybernetik. Frankfurt am Main, Suhrkamp. 196-229.

Pias, C. (2004): Der Auftrag. Kybernetik und Revolution in Chile. In: Gethmann, D./Stauff, M. (Hrsg.): Politiken der Medien. Zürich, Diaphanes. 131-154.

Pias, C. (Hrsg.) (2003): Cybernetics: The Macy-Conferences 1946-1953. Zwei Bände. Berlin Zürich, Diaphanes.

Pohlmann, M. (2018): George Orwell in China: Digitization as a Method of total social control. www.heigos.hypotheses.org/8546

Wang, B./Li, X. (2017): Big Data, Platform Economy and market competition. A preliminary construction of plan-oriented markets economy system in the information age. In: World Review of Political Economy 8/2.

\section{AUTOR + KONTAKT}

Ulrich Petschow ist Mitarbeiter am Forschungsfeld Umweltökonomie und Umweltpolitik am Institut für ökologische Wirtschaftsforschung (IÖW). Institut für ökologische Wirtschaftsforschung (IÖW) GmbH, Potsdamer Str. 105, 10785 Berlin. Tel.: +49 30 884594-0, E-Mail: ulrich.petschow@ioew.de

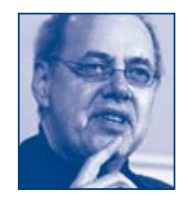

